\title{
Thickness modulation and strain relaxation in strain-compensated \\ InGaP/InGaP multiple-quantum-well structure grown by metalorganic molecular beam epitaxy on GaAs (100) substrate
}

\author{
M. Mitsuhara ${ }^{a, *}$, N. Watanabe ${ }^{a}, H$. Yokoyama $^{a, 1}$, R. Iga ${ }^{a}$, and N. Shigekawa ${ }^{b}$ \\ a NTT Device Technology Laboratories, NTT Corporation, 3-1 Morinosato-Wakamiya, Atsugi,
} Kanagawa 243-0198, Japan

${ }^{\mathrm{b}}$ Graduate School of Engineering, Osaka City University, Osaka 588-8585, Japan

*Corresponding author. E-mail address: mitsuhara.manabu@lab.ntt.co.jp

${ }^{1}$ Present address: National Institute of Technology, Suzuka College, Shiroko-cho, Suzuka, Mie 510-0294, Japan

Keywords: A1. Characterization, A3. Metalorganic molecular beam epitaxy, B1. Phosphides, B2. Semiconducting III-V materials

\begin{abstract}
We have investigated the structural features of a strain-compensated InGaP/InGaP multiple-quantum-well (MQW) structure on GaAs (100) substrate with a band-gap energy of around $1.7 \mathrm{eV}$ for solar cell applications. In transmission electron microscopy images, noticeable thickness modulation was observed in the barrier layers for a sample grown at the
\end{abstract}


substrate temperature of $530^{\circ} \mathrm{C}$. Meanwhile, the X-ray diffraction patterns indicated that strain relaxation predominantly occurred in the well layers. Decreasing the substrate temperature from 530 to $510^{\circ} \mathrm{C}$ was effective in suppressing both the thickness modulation and strain relaxation. Additionally, increasing the growth rate of the well layer further

suppressed the thickness modulation. In room-temperature photoluminescence (PL) emission spectra, the sample grown at $510^{\circ} \mathrm{C}$ showed approximately 50 times higher PL peak intensity than the one grown at $530^{\circ} \mathrm{C}$.

\section{Introduction}

Concentrator solar cells have attracted much attention as the main components of future electricity generation plants using photovoltaic systems [1]. Among the various types of solar cells, the hybrid-tandem solar cell, which is composed of a silicon bottom-cell and a III-V compound top-cell, is an attractive candidate. A hybrid-tandem solar cell possesses both the high mechanical strength of the silicon substrate and the high conversion efficiency of the III-V compound. Recent progress in wafer bonding technologies makes it easy to form bonding interfaces between silicon and III-V compounds with high electrical conductivity [2-4], and series-connected tandem solar cells have been reported [5,6]. In order to obtain high conversion efficiency for a two-junction solar cell containing a silicon bottom-cell with the band-gap of $1.12 \mathrm{eV}$, a band-gap energy from 1.6 to $1.8 \mathrm{eV}$ is preferred for the absorption region of the top-cell [7-9]. Among nearly lattice-matched systems of 
III-V compounds, AlGaAs on GaAs substrate can satisfy this band-gap energy requirement.

Solar cells contain large amounts of III-V material compared with commonly used optoelectronic devices, such as lasers and photodetectors. Therefore, less toxic III-V compounds are preferred as their constituting materials. From this point of view, InGaP on GaAs substrate is promising as a substitute for AlGaAs. This is because InGaP is an arsenic-free material and GaAs substrate can be selectively removed from InGaP with relative ease $[10,11]$. Although the band-gap energy of an InGaP bulk layer is around $1.9 \mathrm{eV}$ under a lattice-matched condition to GaAs, increasing the In content of InGaP to more than 0.6 reduces the band-gap energy to lower than $1.8 \mathrm{eV}$ [12]. In this situation, the lattice-mismatch between $\mathrm{InGaP}$ and $\mathrm{GaAs}$ becomes larger than $+0.8 \%$ and misfit dislocations are easily introduced in the InGaP layer. The strain-compensation technique is effective for suppressing the occurrence of misfit dislocations. However, there are few reports on a strain-compensated multiple-quantum-well (MQW) structure containing compressive-strained InGaP wells.

InGaP QW structures have been applied as the active region of laser diodes used in digital versatile disc (DVD) systems, where InGaAlP is commonly used as a barrier layer to a InGaP well layer in order to ensure the carrier confinement in the wells. In contrast to laser diodes, in an InGaP MQW structure for a solar cell, the photoexcited carriers need to be extracted from the well. Therefore, a material exhibiting a lower barrier height against the well layer is preferable for the barrier layer. Assuming that the strain values of InGaP and InGaAlP barrier layers are the same, the barrier height of an InGaP barrier to a 
compressive-strained InGaP well is lower than that of an InGaAlP barrier. For these reasons, we propose a strain-compensated $\mathrm{InGaP} / \mathrm{InGaP} \mathrm{MQW}$ structure as an absorption region of the III-V top-cell in a hybrid-tandem solar cell.

In this paper, we report on the structural features of the strain-compensated InGaP/InGaP MQWs grown by metalorganic molecular beam epitaxy (MOMBE) on GaAs substrates with a band-gap energy of around $1.7 \mathrm{eV}$. The structural characterization by transmission electron microscopy (TEM) revealed that the thickness modulation predominantly occurred in the InGaP barrier layers. Meanwhile, X-ray diffraction (XRD) patterns indicated large strain relaxation in $\mathrm{InGaP}$ well layers. The thickness modulation and the strain relaxation were suppressed by decreasing the substrate temperature. In addition, increasing the growth rate of the InGaP well layers was effective in further decreasing the thickness modulation. Consequently, the peak intensity and linewidth in photoluminescence (PL) emission spectra could be notably improved by decreasing the substrate temperature and increasing the growth rate of the well layer.

\section{Experimental details}

All samples were grown on Si-doped GaAs (100) just oriented substrates in a modified VG-V90 MOMBE system. Trimethylindium (TMIn), triethylgallium (TEGa), arsine ( $\left.\mathrm{AsH}_{3}\right)$, and phosphine $\left(\mathrm{PH}_{3}\right)$ were the precursors of $\mathrm{In}, \mathrm{Ga}, \mathrm{As}$, and $\mathrm{P}$, respectively. TEGa and TMIn were used without precracking, while $\mathrm{AsH}_{3}$ and $\mathrm{PH}_{3}$ were decomposed in a low-pressure cell 
heated at $900^{\circ} \mathrm{C}$ to produce $\mathrm{As}_{2}$ and $\mathrm{P}_{2}$. These sources were introduced into the growth chamber without a carrier gas. The substrate temperature was monitored with a calibrated optical pyrometer and was varied from 510 to $530^{\circ} \mathrm{C}$.

The samples consisted of a strain-compensated InGaP/InGaP MQW structure sandwiched between nearly lattice-matched InGaP layers with a thickness of about $0.1 \mu \mathrm{m}$. In order to suppress three dimensional growth of strained III-V layers [13], a high group-V source supply (a phosphine beam equivalent pressure of $7.3 \times 10^{-5}$ Torr) was used for the growth of all InGaP layers. The growth rates of the well, barrier, and the lattice-matched InGaP layers for a typical sample grown at $510^{\circ} \mathrm{C}$ were $0.26,0.44$, and $0.35 \mathrm{~nm} / \mathrm{s}$, respectively. For this sample, the strain $\varepsilon_{w}$ and thickness $L_{w}$ of the well layer were $+1.3 \%$ (In content of 0.66 ) and $7.4 \mathrm{~nm}$, and the strain $\varepsilon b$ and thickness $L b$ of the barrier layer were $-0.6 \%$ (In content of 0.41 ) and $12.4 \mathrm{~nm}$. The strains and thicknesses for the well and barrier layers varied with changing the substrate temperature, but the net strain $\varepsilon^{*}$, defined as $\varepsilon^{*}=\left(\varepsilon_{w} L_{w}+\varepsilon b L_{b}\right) /\left(L_{w}+L b\right)$ [14], was within $\pm 0.15 \%$ for all samples in this study.

The structural features of the samples were characterized by TEM observations and XRD measurements. The TEM observations were performed using a HITACHI H-9000 microscope on cross-sectional samples prepared by focused ion beams. The strain and the layer thickness of the MQW region were characterized with a Panalytical X'Pert-MRD high-resolution x-ray diffractometer. The band-gap energy and the material quality were evaluated by room-temperature PL measurements using a laser source operating at a wavelength of $532 \mathrm{~nm}$ and a silicon charge-coupled device (CCD) array. 


\section{Results and discussion}

\subsection{Thickness modulation and strain relaxation}

First, we investigated the impact of the substrate temperature on the structural features of the strain-compensated InGaP/InGaP MQW structure using cross-sectional TEM. For this purpose, samples were grown under the same growth conditions except for the substrate temperature. More specifically, the group-III and group-V source supplies and the growth duration for each layer were fixed. Figures 1(a) and (b) show [011] cross-sectional bright-field TEM images of samples grown at substrate temperatures of 530 and $510^{\circ} \mathrm{C}$. The images were taken with diffraction vector $\mathbf{g}=(200)$. In both images, the dark areas in the MQW regions correspond to the well layers and the bright areas to the barrier layers. The thickness of each layer increased with increasing the substrate temperature from 510 to $530^{\circ} \mathrm{C}$ due to the thermal decompositions of group-III metalorganic sources, but the increases in growth rates were at most $10 \%$. The sample grown at $530^{\circ} \mathrm{C}$ exhibited noticeable thickness modulation in the wells and in the barriers with periods of about $50 \mathrm{~nm}$ in the lateral direction. The difference between the peak and valley heights in an InGaP well or barrier layer increased with increasing number of the periods and reached about $10 \mathrm{~nm}$ at the seventh barrier. The growth of the well layers proceeded so as to cancel the thickness modulation in the barrier layers. Actually, each interface between the first well layer and its upper barrier layer became flat. However, it was difficult for the large valleys in the barrier layers to be buried flatly by the well layers after the first period, and then, threading dislocations along the (111) plane were generated. Note that the thickness modulation can also be seen in InGaP 
layer below the MQW structure for the sample grown at $530^{\circ} \mathrm{C}$, even though this layer is nearly lattice-matched condition to GaAs.

Figure 2 shows the surface morphology of the sample grown at $530^{\circ} \mathrm{C}$ observed using an atomic force microscope (AFM). The AFM image shows elongated features along the (011) direction due to the surface undulation in the (011) direction. Here, the sample surface is InGaP layer above the MQW structure, and the layer thickness is as small as about $0.1 \mu \mathrm{m}$. Therefore, the surface morphology is strongly influenced by the thickness modulation of the uppermost barrier layer. The surface morphology shown in Fig. 2 indicates that the thickness modulation in the MQW structure is formed in the (011) direction.

The thickness modulation could also be seen in the sample grown at $510^{\circ} \mathrm{C}$ as shown in Fig. 1(b), but it evolved more slowly in comparison with that in the sample grown at $530^{\circ} \mathrm{C}$. Specifically, the sixth well of the sample grown at $510^{\circ} \mathrm{C}$ has a similar cross-sectional shape to the second well of the sample grown at $530^{\circ} \mathrm{C}$, and the difference between the peak and valley heights is no more than $3 \mathrm{~nm}$ even at the upper part of the MQW. As a result, the interfaces between the well and the barrier layers in the sample grown at $510^{\circ} \mathrm{C}$ were much smoother than in the sample grown at $530^{\circ} \mathrm{C}$. In the sample grown at $510^{\circ} \mathrm{C}$, no crystalline defects could be seen in the observation area of the cross-sectional TEM, which had a length of $1.8 \mu \mathrm{m}$ in the lateral direction (not shown in the figure). In summary, a comparison of Figs. 1(a) and (b) reveals that decreasing the substrate temperature is effective in suppressing the thickness modulation in the strain-compensated InGaP/InGaP MQW structure.

The anisotropic thickness modulation mentioned above has already been reported for 
strain-compensated MQW structures of other material systems, such as $\operatorname{InGaAs}(\mathrm{P})$ and $\operatorname{InAsP}$ [15-17]. As in those previous studies, growth of the compressive-strained well layers is more favorable on locally thin regions of the tensile-strained barrier layer, and also the thickness modulation can be suppressed by decreasing the substrate temperature $[18,19] . \quad$ In contrast, for the other material systems, a compressive strain larger than $1.5 \%$ is usually required to induce the thickness modulation in the MQW region within a small number of periods $[17,20] . \quad$ In contrast, in this study, even though the compressive strain of InGaP well is at most $1.3 \%$, the thickness modulation begins to occur at an early stage of the MQW growth as shown in Fig. 1(a). It has been reported that the surface of InGaP tends to undulate easily even under a lattice-matched condition to GaAs [21, 22], as seen in Fig.1(a). The morphological instability of InGaP will affect the thickness modulation in strain-compensated InGaP/InGaP MQW structure.

Next, we evaluated the effect of the substrate temperature on the structure of the MQW by analyzing the XRD patterns. Figure 3 shows the experimental and simulated XRD patterns using the (400) reflection of the samples grown at substrate temperatures of 530, 520, and $510^{\circ} \mathrm{C}$. The detailed simulation methods and results are described in the following paragraph. The positions indicated by arrows are the angles of the zeroth-order satellite peaks corresponding to the average lattice constants for each MQW. The positions marked with triangles correspond to the InGaP layers above and below the MQW structure. Broad satellite peaks with large background counts could be seen in the XRD patterns for the sample grown at $530^{\circ} \mathrm{C}$, while the satellite peaks became narrower as the substrate temperature 
decreased from 530 to $510^{\circ} \mathrm{C}$. This means that the vertical periodicity of the MQW structure is improved by decreasing the substrate temperature. As for the position of the satellite peaks, the peaks at the lower-angle side of GaAs shifted to the lower angle with decreasing substrate temperature, while the peaks at the higher-angle side stayed at nearly the same angle. Here, the positions of the envelope functions of the satellite peaks at the lower and the higher angle side of GaAs correspond to the out-of-plane lattice constants of the well and barrier layers, respectively. Therefore, the out-of-plane lattice-constant of the well layer, in comparison with that of the barrier layer, was strongly affected by the variation of the substrate temperature.

For a fixed composition of InGaP, the strain relaxation can be evaluated by comparing experimental XRD pattern with simulated one. However, because increasing the substrate temperature promotes $\mathrm{Ga}$ incorporation into InGaP layer in the MOMBE growth [23], the highly-accurate composition of InGaP layer is difficult to determine. Therefore, we tried to perform the simulation fitting of XRD pattern without considering the strain relaxation. Specifically, we estimated the strains in the well and barrier layers using the dynamical simulation [24], assuming that both layers are pseudomorphically strained with the in-plane lattice constant of GaAs. The simulated XRD patterns are shown in Fig. 3. Decreasing the substrate temperature from 530 to $510^{\circ} \mathrm{C}$ leads to a better agreement between the experimental and simulated patterns. This result indicates that the strain relaxation was suppressed by decreasing the substrate temperature. Figure 4 shows the strains in the well and barrier layers used in the simulation versus the substrate temperature. The well layer 
strain shifted from +1.3 to $+0.7 \%$ with increasing substrate temperature from 510 to $530^{\circ} \mathrm{C}$.

In contrast, the barrier layer strain remained almost constant at $-0.6 \%$ against the variation of substrate temperature. In the MOMBE growth of InGaP, it has been reported that the $\mathrm{Ga}$ content significantly increases with increasing substrate temperature from 490 to $590^{\circ} \mathrm{C}$ [23]. However, the increase in the substrate temperature was no more than $20^{\circ} \mathrm{C}$ in this study, and the peak shifts for InGaP layers above and below the MQW structure were at most 0.33 degrees, as indicated by the triangles in Fig. 3. In addition, all three samples in Fig. 3 have the PL emission peaks at around $1.7 \mathrm{eV}$ as will be shown later. These results indicate that a variation of $\mathrm{Ga}$ content of InGaP well layer among the sample grown at 510,520 , and $530^{\circ} \mathrm{C}$ is not large. That is to say, the strain relaxation due to increasing the substrate temperature is more significant in the well layers than in the barrier layers.

\subsection{Compositional modulation}

Now, let us consider the likely causes of the thickness modulation in the strain-compensated InGaP/InGaP MQW structure. Several thickness modulation mechanisms, such as $\mathrm{CuPt}$ ordering and lateral compositional modulation, have been proposed [21]. Among the possible mechanisms, lateral compositional modulation can be examined by cross-sectional TEM observation using the diffraction vector with a vector component perpendicular to the growth direction $[15,20,22]$. Figures $5(a)$ and (b) show the [011] cross-sectional TEM images taken with diffraction vector $\mathbf{g}=(022)$ for samples grown at substrate temperatures of 530 and $510^{\circ} \mathrm{C}$. For these two samples, the TEM images taken 
with diffraction vector $\mathbf{g}=(200)$ have already been shown in Figs. 1(a) and (b), respectively. As shown in Figs. 5(a) and (b), the variation in the contrast along the (011) direction can be clearly seen in both TEM images. The contrast variations indicate the existence of lateral compositional modulations of the In and $\mathrm{Ga}$ in the barrier and well layers. Therefore, the lateral compositional modulation seems to be one of the probable cause of the thickness modulation.

At the end of the section, we briefly comment on the effect of the CuPt ordering on the thickness modulation in this study. It is well known that the $\mathrm{CuPt}$ ordering in $\mathrm{InGaP}$ is highly dependent on the growth condition and the ordering reduces the band-gap energy from 1.9 to $1.85 \mathrm{eV}$ under a lattice-matching condition to GaAs [25]. In our investigation of InGaP grown by MOMBE, the band-gap energy determined by the PL peak position of InGaP lattice-matched to GaAs was about $1.9 \mathrm{eV}$ (not shown in the figure). This result suggests that the CuPt ordering in InGaP has little effect on the thickness modulation in this study.

\subsection{Influence of growth rate}

In our previous study, we have reported that strain relaxation in compressive-strained InGaAs- or InAsP-MQW on InP substrate is closely related to the thickness modulation [20, 26, 27]. An effective way to suppress the strain relaxation in a compressive-strained layer is to increase the growth rate [27-29]. Next, we investigated the effect of the growth rate of the well layer on the structural features of the strain-compensated InGaP/InGaP MQW. We prepared a sample grown at the substrate temperature of $510^{\circ} \mathrm{C}$ with high growth rate of the 
well layer. The growth rate of the well layer increased from 0.26 to $0.43 \mathrm{~nm} / \mathrm{s}$, while the strain and the thickness were nearly equal to those of the sample grown at $510^{\circ} \mathrm{C}$ in Fig. 3 . The same barrier-layer growth conditions were used, while the source-gas supplies for InGaP layers above and below the MQW structure were adjusted to the lattice-matched condition to GaAs. Figure 6 shows the experimental and simulated XRD patterns of the sample. We performed the simulation of the XRD pattern, assuming that the strain and thickness of the InGaP well layer were $+1.3 \%$ and $7.5 \mathrm{~nm}$ and those of the barrier layer were $-0.6 \%$ and 12.4 nm. As can be seen in the experimental XRD pattern, the satellite peaks of the sample with high growth rate of the well layer are more clearly defined than those of the sample grown at $510^{\circ} \mathrm{C}$ with the low growth rate in Fig. 3. In addition, the experimental XRD pattern agrees well with the simulated one. This result means that the uniformity of the MQW structure is improved by increasing the growth rate of the well layer. To investigate the thickness modulation in the MQW structure, we performed a TEM observation. Figure 7 shows a $[0 \overline{1}$ 1] cross-sectional TEM image of the sample taken with diffraction vector $\mathbf{g}=(200)$. The thickness modulation of the barrier layers can still be seen in the sample with the high-growth-rate well layer, but the difference between the peak and valley heights in an InGaP barrier layer is considerably smaller than that in the sample with the low growth rate in Fig. 1(b). Accordingly, the XRD satellite peaks for the sample grown with the high growth rate shown in Fig. 6 are narrower than those for the sample grown with the low growth rate shown in Fig. 3. 


\subsection{Photoluminescence properties}

Finally, we investigated the PL properties of the strain-compensated $\mathrm{InGaP} / \mathrm{InGaP}$ MQWs in order to evaluate the band-gap energies and the material quality of the samples. The measured samples were grown at substrate temperatures of 530,520 , and $510^{\circ} \mathrm{C}$ with low well-layer growth rates $[0.26-0.27 \mathrm{~nm} / \mathrm{s}]$ and at $510^{\circ} \mathrm{C}$ with a high well-layer growth rate $[0.43 \mathrm{~nm} / \mathrm{s}]$. The XRD patterns of the samples have already been shown in Figs. 3 and 6 . Figure 8 compares the room-temperature PL emission spectra of the samples. For each spectrum, the positions marked with triangles correspond to the PL emission peaks from the InGaP wells. The PL emission energies were around $1.7 \mathrm{eV}$, and there was not much difference between the samples. On the other hand, the PL peak intensity noticeably increased with decreasing substrate temperature from 530 to $510^{\circ} \mathrm{C}$, as shown in the upper three spectra in Fig. 8. Specifically, the peak intensity for the sample grown at $510^{\circ} \mathrm{C}$ was approximately 50 times higher than that for the sample grown at $530^{\circ} \mathrm{C}$. As shown in the TEM images in Figs. 1(a) and (b), the generation of crystalline defects can be suppressed by decreasing the substrate temperature. Therefore, the high PL intensity for the sample grown at $510^{\circ} \mathrm{C}$ is due to the low defect density. In contrast, increasing the InGaP well growth rate from 0.26 to $0.43 \mathrm{~nm} / \mathrm{s}$ had a relatively low impact on the PL peak intensity, as can be seen from the lower two spectra in Fig. 8. However, the full width at half maximum (FWHM) of the PL emission spectrum substantially decreased from 90 to $61 \mathrm{meV}$ with increasing well-layer growth rate. These results can be understood by comparing the cross-sectional TEM images of the two samples in Fig. 1(b) and Fig. 7. No threading dislocations are seen 
in the two samples grown at $510^{\circ} \mathrm{C}$. Therefore, the difference in defect density between the samples can be considered to be small. Meanwhile, the thickness modulations not only in the barrier but also in the well layers were suppressed by increasing the well-layer growth rate. It is known that the thickness modulation in a quantum well results in an increase of the linewidth in PL emission spectrum [30]. Therefore, the decrease of the FWHM value in the PL spectrum can be attributed to the suppression of the thickness modulation in the InGaP well layers.

\section{Conclusion}

We studied the structural features and optical properties of strain-compensated InGaP/InGaP MQWs grown on GaAs substrates, which is attractive as an arsenic-free top-cell material for hybrid-tandem solar cells with a silicon bottom cell. Thickness modulation in the MQW structure became noticeable with increasing both substrate temperature and the number of periods. A cross-sectional TEM image taken with diffraction vector $\mathbf{g}=(022)$ revealed that compositional modulation in $\mathrm{InGaP}$ was the likely cause of the thickness modulation. On the other hand, an analysis of the XRD patterns indicated noticeable strain relaxation in the well layer. Both the thickness modulation and strain relaxation could be suppressed by decreasing the substrate temperature from 530 to $510^{\circ} \mathrm{C}$. Increasing the growth rate of the well layer was effective in further suppressing the thickness modulation. The peak energies of the room-temperature PL emission spectra for the MQWs were around 
$1.7 \mathrm{eV}$ regardless of the substrate temperature. The peak intensity and linewidth in PL emission spectrum were significantly improved both by decreasing the substrate temperature and increasing the well-layer growth rate. These findings show that crystalline degradation of the $\mathrm{InGaP} / \mathrm{InGaP} \mathrm{MQW}$ structure is caused by thickness modulation and strain relaxation and that both lowering the substrate temperature and increasing the well-layer growth rate are effective for suppressing the degradation.

\section{Acknowledgement}

We thank R. Sato and S. Mizuno for the TEM observations, and Y. Oiso, M. Arai, and T. Hoshi for fruitful discussions. We also acknowledge H. Matsuzaki, M. Ito, M. Kohtoku, H. Oohashi, and S. Kodama for their encouragement. This work was supported in part by the "Creative Research for Clean Energy Generation Using Solar Energy" project in the Core Research for Evolutionary Science and Technology (CREST) programs of the Japan Science and Technology Agency (JST). 


\section{References}

[1] M. Yamaguchi, Energy Procedia, 15 (2012) 265.

[2] M. Howlader, T. Watanabe, and T. Suga, J. Appl. Phys. 99 (2002) 3062.

[3] S. Essig, O. Moutanabbir, A. Wekkeli, H. Nahme, E. Oliva, A. Bett, and F. Dimroth, J. Appl. Phys. 113 (2013) 203512.

[4] J. Liang, S. Nishida, M. Morimoto, and N. Shigekawa, Electron. Lett. 49 (2013) 830.

[5] K. Derendorf, S. Essig, E. Oliva, V. Klinger, T. Roesener, S. P. Philipps, J. Benick, M. Hermle, M. Schachtner, G. Siefer, W. Jager, and F. Dimroth, IEEE J. Photovoltaics 3 (2013) 1423.

[6] K. Tanabe, K. Watanabe, and Y. Arakawa, Scientific Reports 2:349 (2012) 1.

[7] F. Dimroth, U. Schubert, and A. W. Bett, IEEE Electron Device Lett. 21 (2000) 209.

[8] S. Kurtz, P. Faine, and J. Olson, J. Appl. Phys. 68 (1990) 1890.

[9] M. E. Nell and A. M. Barnett, IEEE Trans. Electron Devices ED-34 (1987) 257.

[10] A. R. Clawson, Mater. Sci. Eng. A31 (2001) 1.

[11] K. Lee, J. D. Zimmerman, X. Xiao, K. Sun, and S. R. Forrest, J. Appl. Phys. 111 (2012) 033527.

[12] I. Vurgaftman, J. Meyer, and L. Ram-Mohan, J. Appl. Phys. 111 (2001) 5815.

[13] J.-Y. Emery, C. Starck, L. Goldstein, A. Ponchet, and A. Rocher, J. Cryst. Growth 127 (1993) 241.

[14] M. Ogasawara, H. Sugiura, M. Mitsuhara, M. Yamamoto, and M. Nakao, J. Appl. Phys. 84 (1998) 4775. 
[15] J. Ponchet, A. Rocher, J.-Y. Emery, C. Starck, and L. Goldstein, J. Appl. Phys. 74 (1993) 3778.

[16] L. Nasi, C. Ferrari, A. Lanzi, L. L. Lazzarini, R. Balboni, G. Clarke, M. Mazzer, C. Rohr, P. Abbott, and K. Barnham, J. Cryst. Growth 274 (2005) 65.

[17] J. Ponchet, A. Rocher, J.-Y. Emery, C. Starck, and L. Goldstein, J. Appl. Phys. 77 (1995) 1977.

[18] A. Smith, A. Briggs, K. Scarrott, X. Zhou, and U. Bangert, Appl. Phys. 65 (1994) 2311.

[19] P. Desjardins, H. Marchand, L. Isnard, and R. A. Masut, J. Appl. Phys. 81 (1997) 3501.

[20] M. Mitsuhara, M. Ogasawara, and H. Sugiura, J. Cryst. Growth 210 (2000) 463.

[21] M. Schmidbauer, A. Ugur, C. Wollstein, F. Hatami, F. Katmis, O. Caha, and W. T. Masselink, J. Appl. Phys. 111 (2012) 024306.

[22] Y. Wang, Z.Wang, T. Brown, A. Brown, and G. May, J. Electron. Mater. 29 (2000) 1372.

[23] J. Garcia, P. Maurel, P. Bove, and J. Hirtz, J. Appl. Phys. 69 (1991) 3297.

[24] Panalytical X'pert Epitaxy simulation software Ver. 4.1, 2004.

[25] A. Gomyo, T. Suzuki, K. Kobayashi, S. Kawata, and I. Hino, Appl. Phys. Lette. 50 (1987) 673.

[26] M. Mitsuhara, M. Ogasawara, M. Oishi, and H. Sugiura, Appl. Phys. Lett. 72 (1998) 3106.

[27] M. Ogasawara, M. Mitsuhara, M. Itoh, and T. Amano, J. Cryst. Growth 205 (1999) 489.

[28] N. Grandjean, J. Massies, and M. Leroux, J. Leymarie, A. Vasson, and A. M. Vasson, 
Appl. Phys. Lett. 64 (1994) 2664.

[29] S. Kim, J. Kirch, and L. Mawst, J. Cryst. Growth 312 (2010) 1388-1390.

[30] L. Goldstein, Y. Horikoshi, S. Tarucha, and H. Okamoto, Jpn. J. Appl. Phys. 22 (1983) 1489. 


\section{Figure captions}

Figure 1.

Cross-sectional TEM images using diffraction vector $\mathbf{g}=(200)$ for samples grown at substrate temperatures of (a) 530 and (b) $510^{\circ} \mathrm{C}$. Dark and bright areas in the MQW region correspond to the well and barrier layers, respectively.

Figure 2.

$2 \mu \mathrm{m} \times 2 \mu \mathrm{m}$ AFM image of the sample grown at the substrate temperature of $530^{\circ} \mathrm{C}$. Cross-sectional TEM image of the sample is shown in Fig. 1(a).

Figure 3.

Experimental XRD patterns around the symmetric (400) reflection for samples grown at different substrate temperatures. Thick and thin lines correspond to the experimental and simulated patterns, respectively.

Figure 4.

Plots of the strains in the well and barrier layers used in the simulation of XRD pattern shown in Fig. 3 versus substrate temperature. The lines are guides for the eye.

Figure 5.

Cross-sectional TEM images using diffraction vector $\mathbf{g}=(022)$ for samples grown at substrate 
temperatures of (a) 530 and (b) $510^{\circ} \mathrm{C}$. The TEM images using diffraction vector $\mathbf{g}=(200)$ are shown in Fig. 1.

Figure 6.

Experimental and simulated XRD patterns around the symmetric (400) reflection for the sample grown at substrate temperature of $510^{\circ} \mathrm{C}$ with a high well-layer growth rate $(0.43$ $\mathrm{nm} / \mathrm{s})$.

Figure 7.

Cross-sectional TEM image using diffraction vector $\mathbf{g}=(200)$ for the sample grown at substrate temperature of $510^{\circ} \mathrm{C}$ with a high well-layer growth rate $(0.43 \mathrm{~nm} / \mathrm{s})$. Dark and bright areas in the MQW region correspond to the well and barrier layers, respectively.

Figure 8.

Room-temperature PL spectra of MQWs grown at substrate temperatures of 530, 520, and $510^{\circ} \mathrm{C}$ with low well-layer growth rates $(0.26-0.27 \mathrm{~nm} / \mathrm{s})$ and at $510^{\circ} \mathrm{C}$ with a high growth rate $(0.43 \mathrm{~nm} / \mathrm{s})$. 


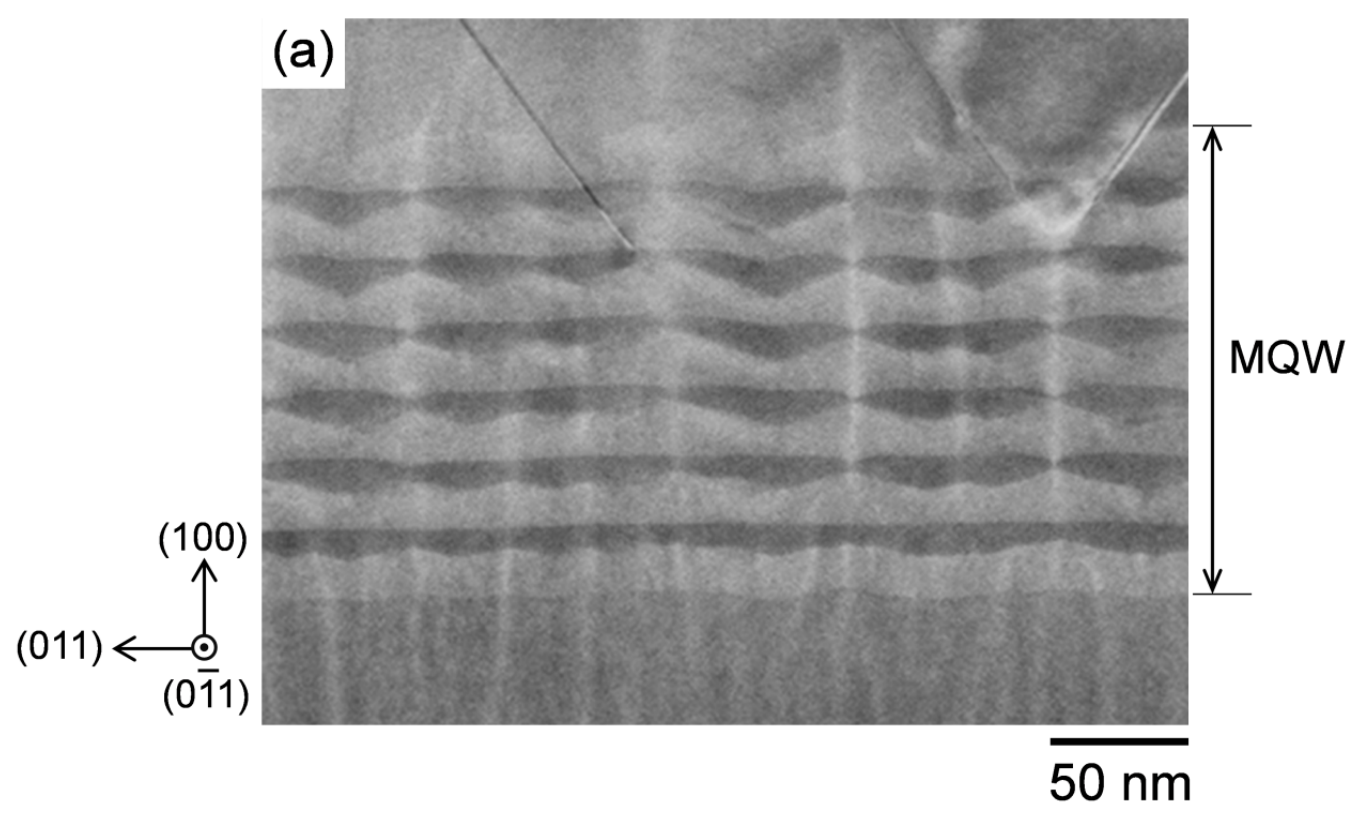

(b)

Figure 1 


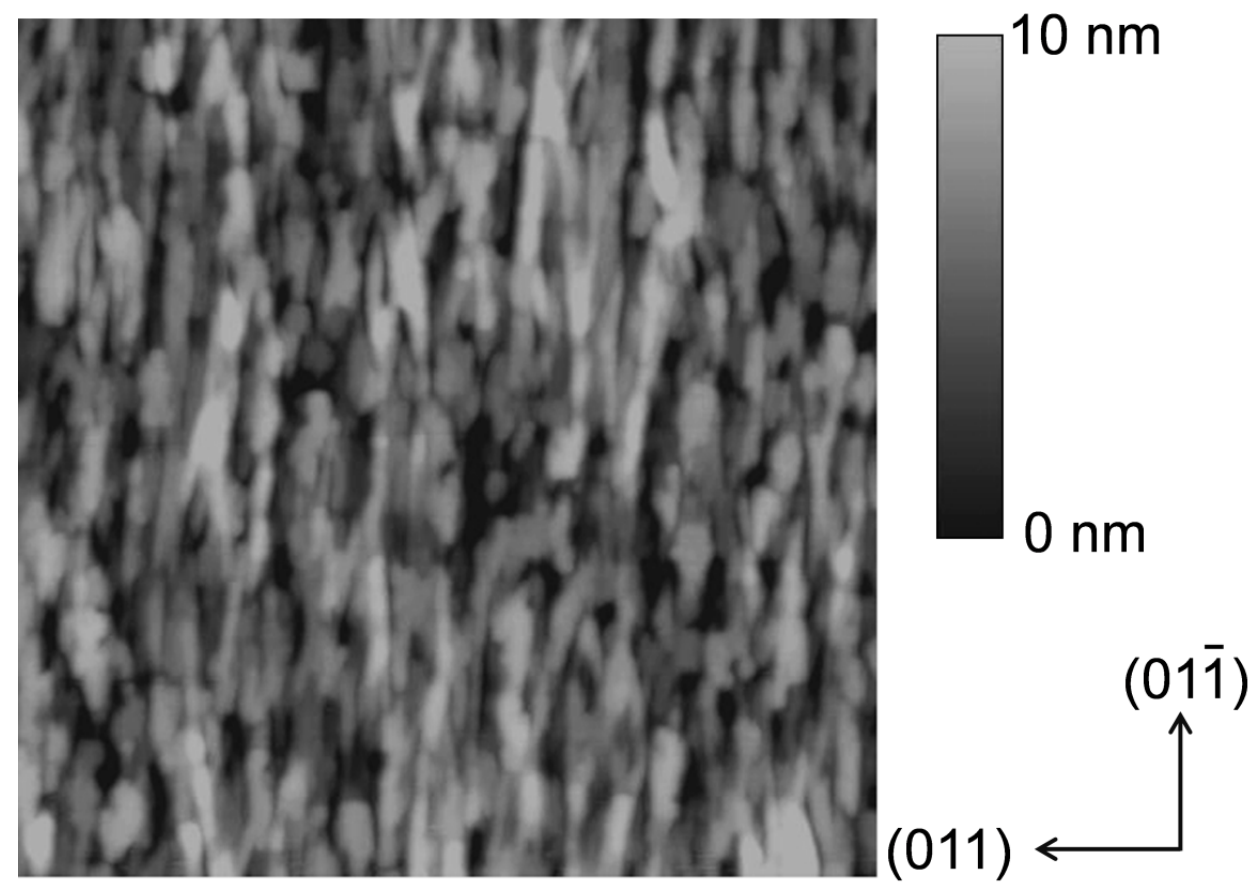

Figure 2 


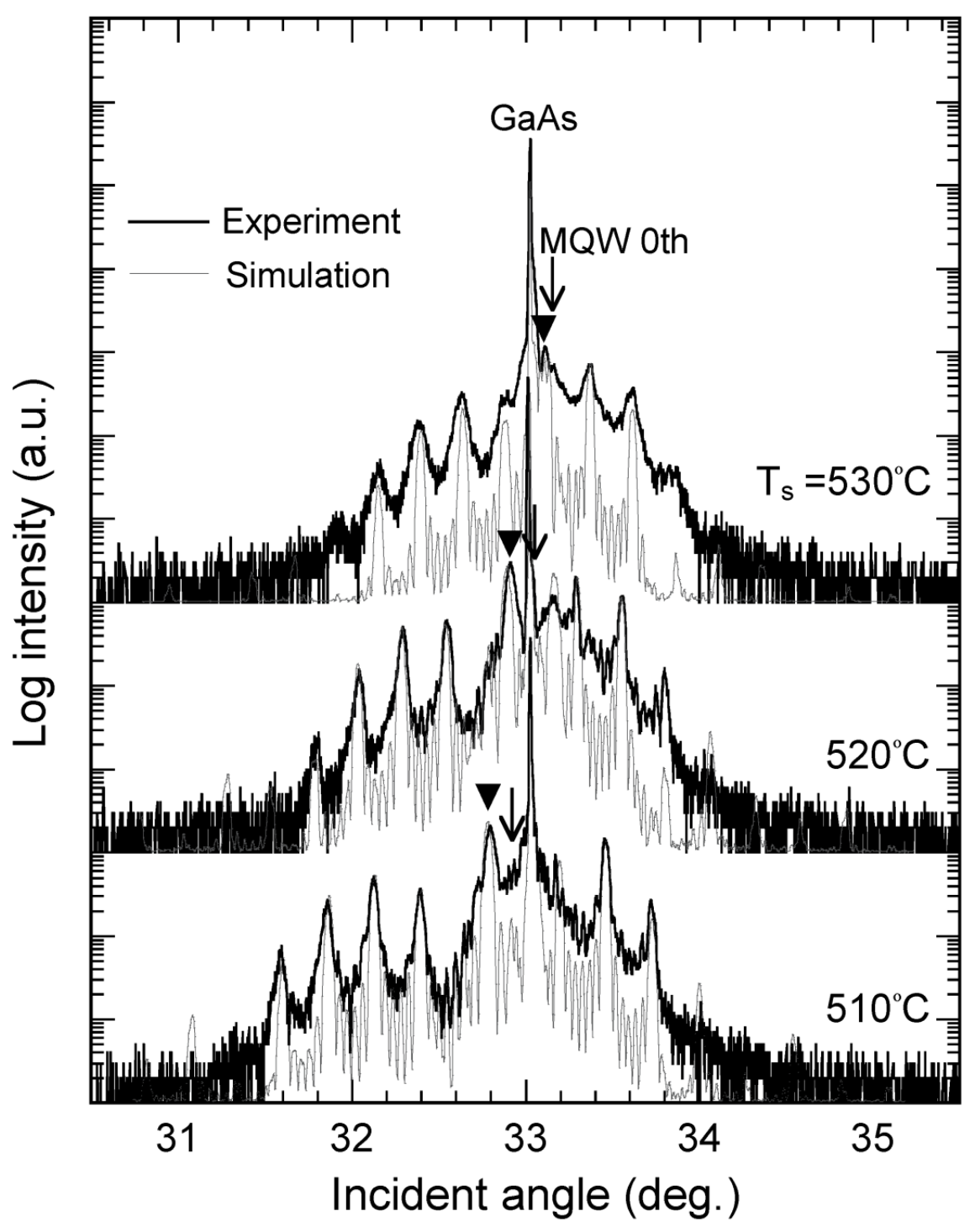

Figure 3 


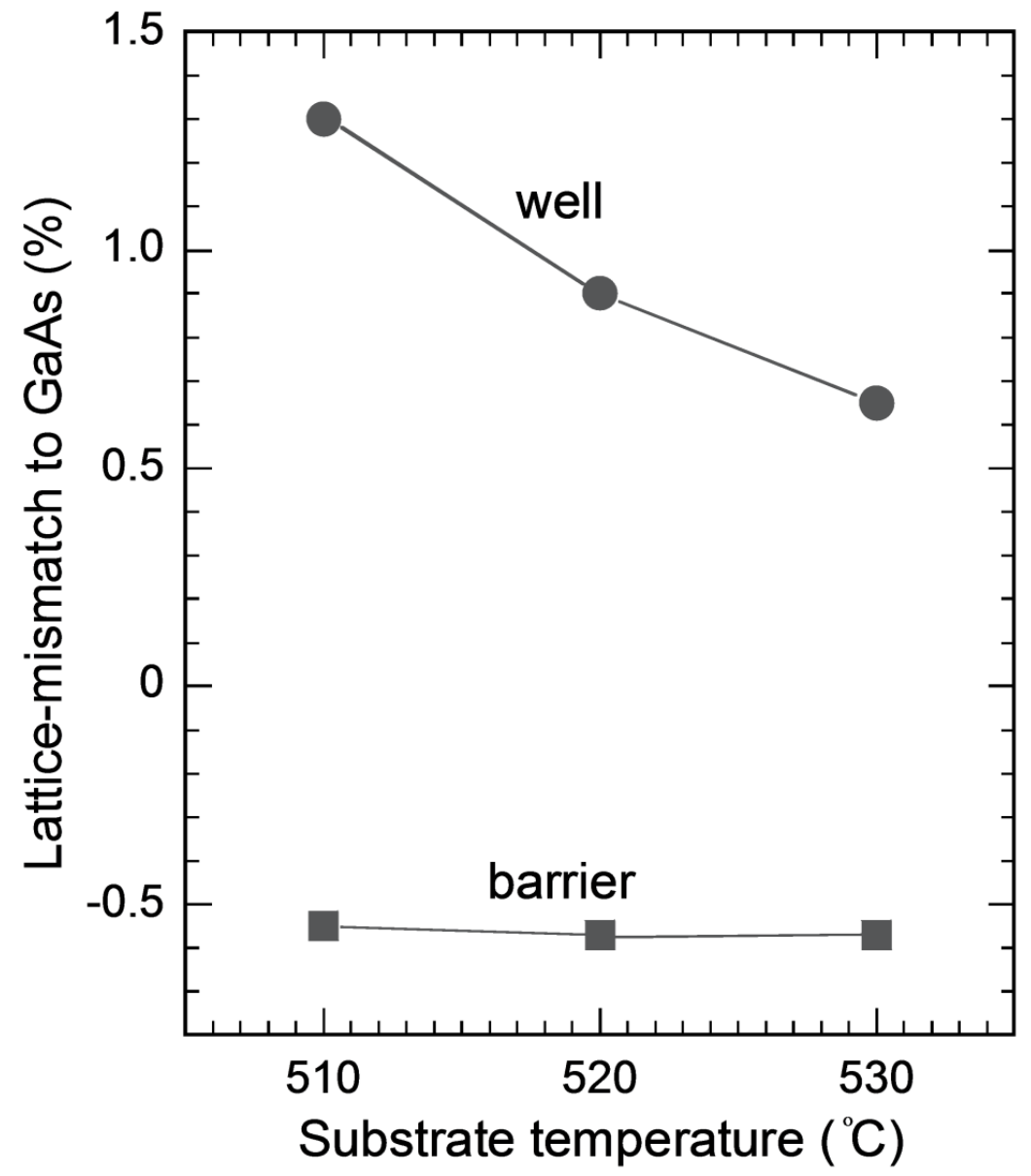

Figure 4 

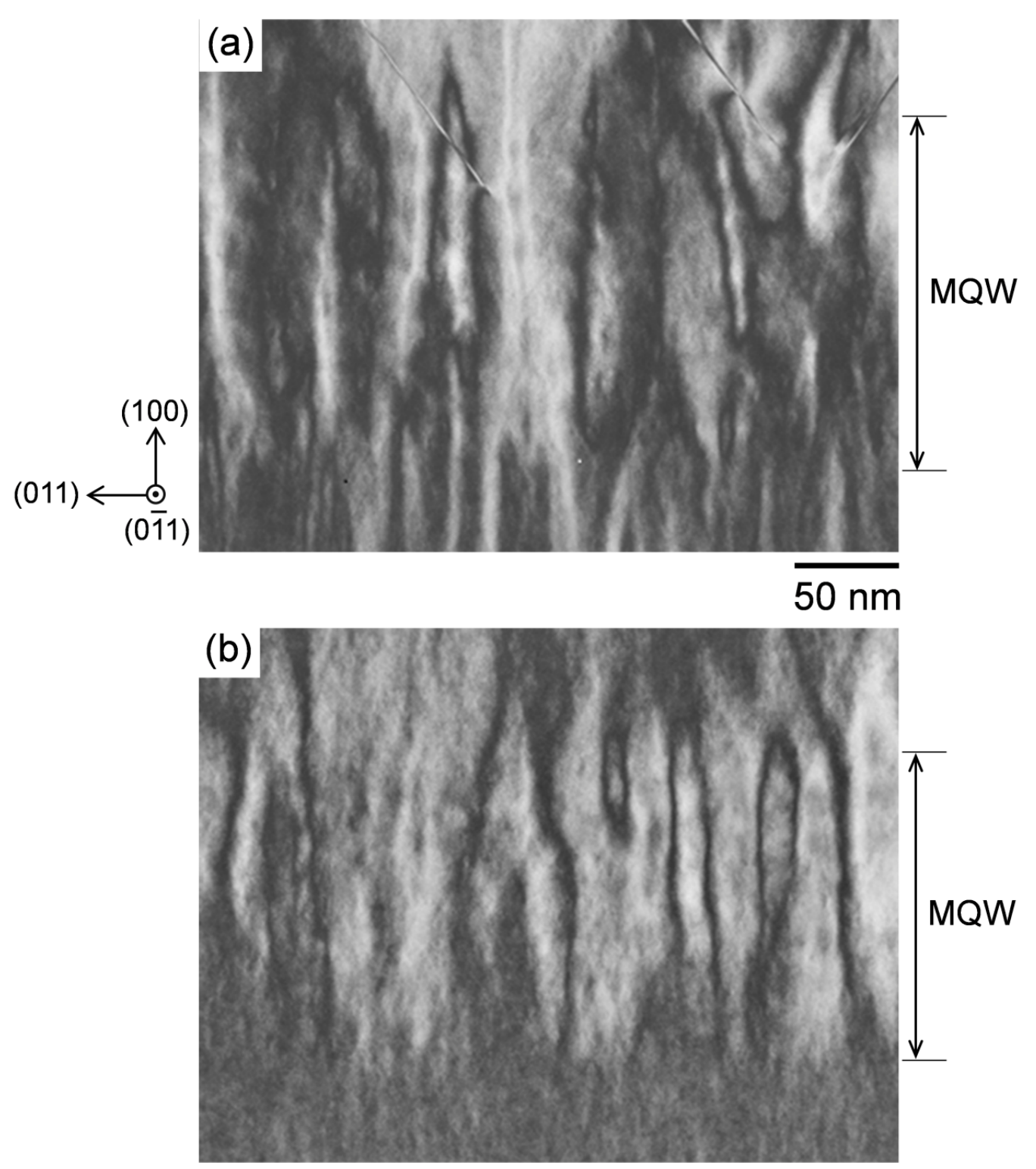

Figure 5 


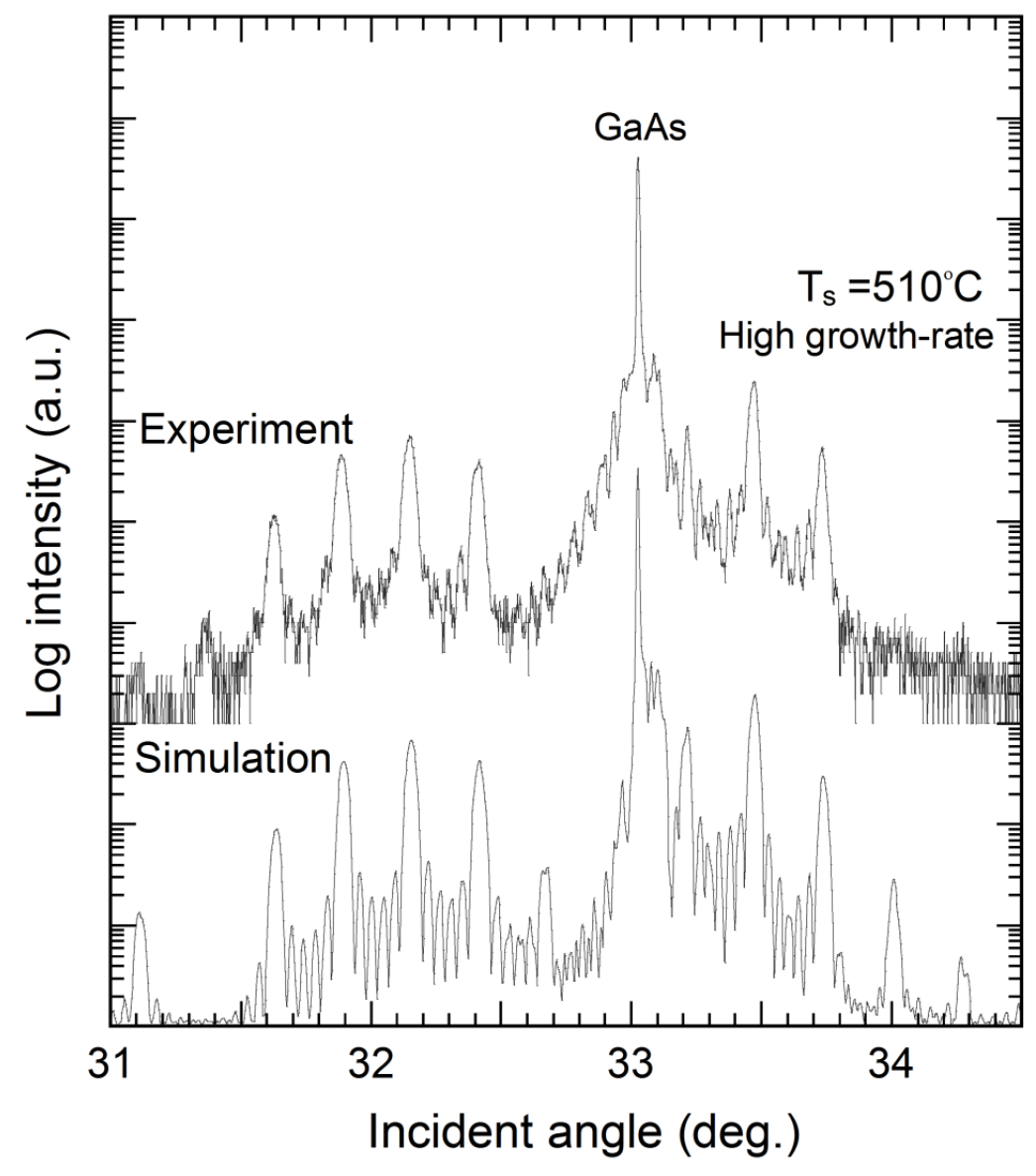

Figure 6 


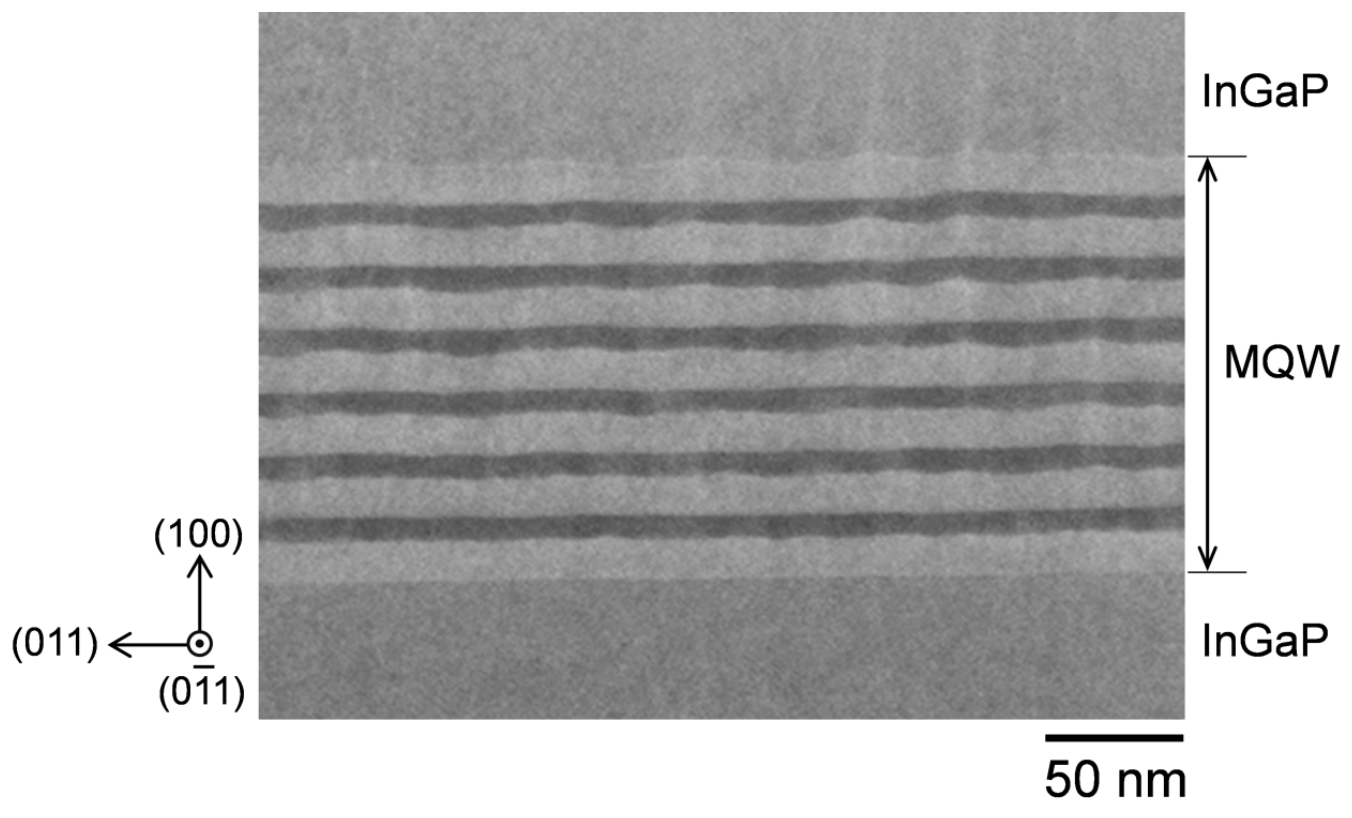

Figure 7 


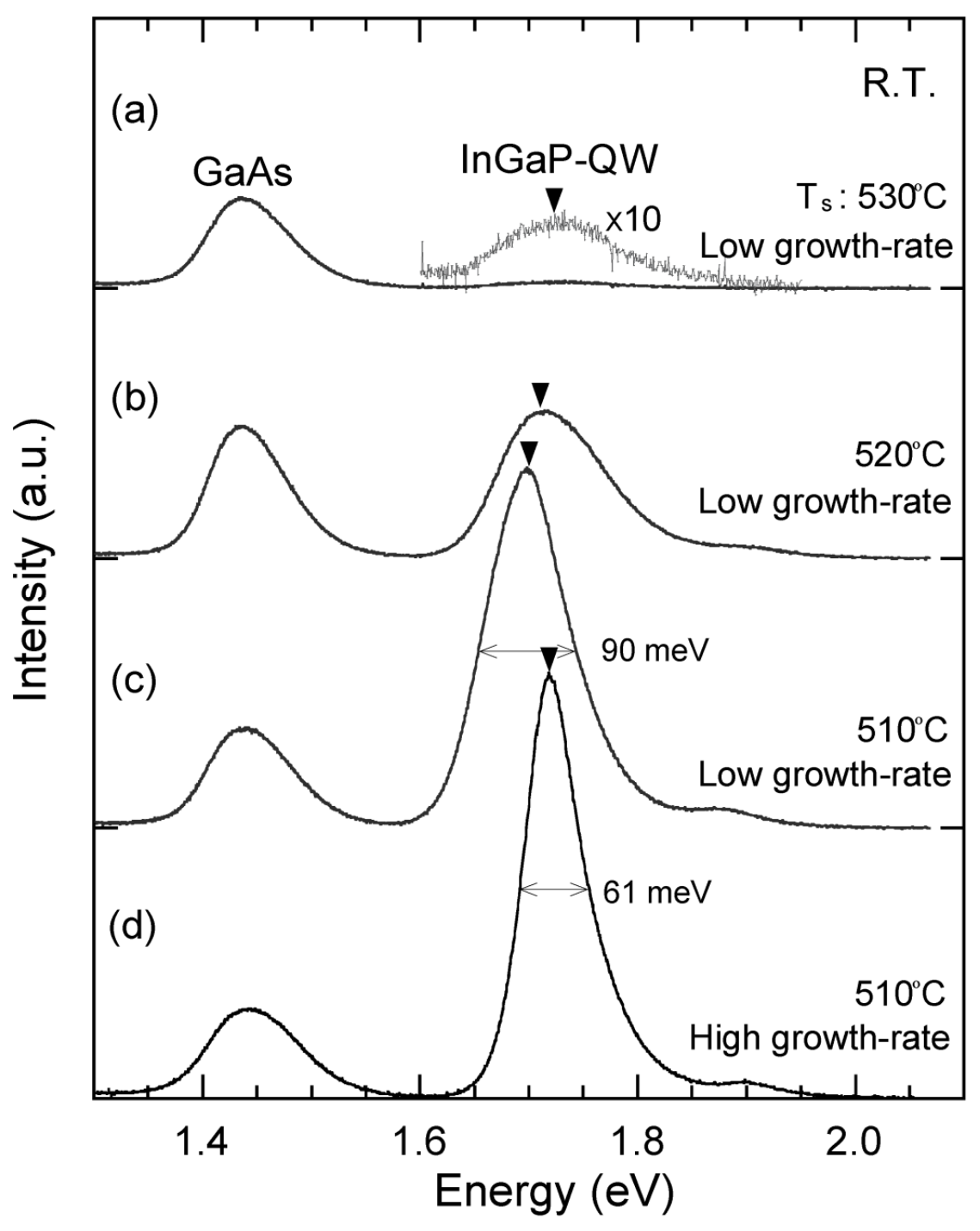

Figure 8 